\title{
Joint terminals and relay optimization for two-way power line information exchange systems with QoS constraints
}

\author{
Xiaolin $\mathrm{Wu}^{*}$ and Yue Rong
}

\begin{abstract}
The quality-of-service (QoS) criteria (measured in terms of the minimum capacity requirement in this paper) are very important to practical indoor power line communication (PLC) applications as they greatly affect the user experience. With a two-way multicarrier relay configuration, in this paper we investigate the joint terminals and relay power optimization for the indoor broadband PLC environment, where the relay node works in the amplify-and-forward (AF) mode. As the QoS-constrained power allocation problem is highly non-convex, the globally optimal solution is computationally intractable to obtain. To overcome this challenge, we propose an alternating optimization (AO) method to decompose this problem into three convex/quasi-convex sub-problems. Simulation results demonstrate the fast convergence of the proposed algorithm under practical PLC channel conditions. Compared with the conventional bidirectional direct transmission (BDT) system, the relay-assisted two-way information exchange (R2WX) scheme can meet the same QoS requirement with less total power consumption.
\end{abstract}

Keywords: Power allocation; Power line communication (PLC); Two-way information exchange; Non-regenerative relay; Quality-of-service (QoS)

\section{Introduction}

With the emerging concept of smart grid, communication infrastructures used in the power grid have received much research interest recently. Compared with other communication systems, power line communication (PLC) technology has been considered as a competitive candidate for smart grid for its unique advantage-power cables are preexisting almost in every town of the world. In smart grid, the power cables are used as medium, in addition to electricity delivery, to support information exchanges between appliances, power meters, and/or transformers [1].

As reported in [2], there are certain similarities between PLC and wireless transmission characteristics, for example the frequency-selective nature of the propagation channel. Thus, many signal processing techniques which have been developed for wireless communication systems can be adopted into PLC, such as compressed sensing [3],

*Correspondence: xiaolin.wu@curtin.edu.au

Department of Electrical and Computer Engineering, Curtin University, Bentley, WA, Australia cognitive radio [4], and multiple-input multiple-output (MIMO) techniques $[5,6]$. In particular, based on the common broadcasting nature between the wireless signal propagation and the power-cable-guided signal transmission, research on the application of modern wireless relaying processing for PLC has become an attractive topic. In [7], the author described a relay-assisted PLC system based on single-frequency networking condition. The authors of [8] investigated the optimal time duration allocation between the direct transmission phase and the relaying phase, along with the optimal power allocation problem, when the relay node works in an opportunistic manner. Under the assumption that each outlet on the power grid is a potential relay node, the authors of [9] proposed a multi-hop transmission scheme combined with the application of distributed space-time block code (DSTBC) in PLC networks.

Depending on the signal processing used at the relay node, relay transmission systems can be classified into two groups, namely regenerative and non-regenerative relay systems. In the regenerative relay scheme, the relay node decodes the received signal firstly and then recodes and

\section{Springer}

(C) 2015 Wu and Rong. Open Access This article is distributed under the terms of the Creative Commons Attribution 4.0 International License (http://creativecommons.org/licenses/by/4.0/), which permits unrestricted use, distribution, and reproduction in any medium, provided you give appropriate credit to the original author(s) and the source, provide a link to the Creative Commons license, and indicate if changes were made. 
forwards it to the destination node. This technique is also called the decode-and-forward (DF) relaying. On the other hand, the non-regenerative scheme only requires the relay node to amplify-and-forward (AF) its received signal. Thus, the AF relay has lower complexity, shorter processing delay, and lower implementation cost.

In some applications such as remote meter reading, two PLC terminal nodes need to exchange information with the aid of a relay node. In conventional half-duplex relay systems, where the relay node cannot transmit and receive simultaneously at the same time and frequency, four timefrequency slots are required for a two-way information exchange [10]. To improve the system spectral efficiency, the authors of [11] proposed the analog network coding technique, where two-way information exchange can be completed in two time-frequency slots with a practical half-duplex relay node. We call the system in [11] a two-way relay system, which was firstly investigated under wireless environments. The authors of [12] considered an AF scheme for two-way relaying over orthogonal frequency-division multiplexing (OFDM) system. They performed power allocation at the relay and both terminals, as well as tone permutation at the relay node, in order to maximize the sum capacity. The authors of [13] introduced the idea of two-way relay scheme into PLC scenario, and this scheme has been addressed in detail in a recent work [14]. In [14], the authors considered that a relay device, working in the AF mode, is used to assist the data exchange of two terminals in an indoor PLC network, and found that the AF two-way system can increase the achievable data exchange rate.

In the conventional one-way direct transmission (DT) system, the rate maximization (RM) and margin maximization (MM) problems are of duality to each other and admit a unique water-filling solution [15]. However, this fact does not hold when a relay node has been introduced. The aim of [12-14] is to maximize a given objective function, for example signal-to-noise ratio (SNR), mutual information (MI), or system capacity, subjecting to the power constraints of the whole network or/and at each node. However, the quality-of-service (QoS) constraints are not addressed. Note that in practical indoor PLC applications, such as high definition video streaming, QoS criteria are very important as they greatly affect the user experience.

In this paper, we adopt the two-way non-regenerative multicarrier relay scheme for indoor PLC networks with QoS considerations. In particular, we consider the joint optimization of the power allocation of two terminals and the relay node to minimize the network transmission power consumption subjecting to QoS requirements, which has not been considered before. We mention that in practical PLC systems, smaller transmission power leads to lower electromagnetic interference (EMI) to other nearby communication systems. We set the QoS criteria as the lower-bounds of the capacity of the data traffic from one terminal to the other. Since this QoS-constrained power allocation problem is highly non-convex, the globally optimal solution is computationally intractable to obtain. To overcome this challenge, we propose an alternating optimization ( $\mathrm{AO}$ ) method adopted from [16] to decompose the joint optimization problem into three subproblems. Simulation results show the fast convergence of the proposed algorithm.

The remainder of this paper is organized as follows. Section 2 describes the indoor power grid topology. Following this, we present in detail the system model and problem formulation in Section 3. The problem formulated is a joint optimization of power allocation between two terminals and one relay node, subjecting to minimum capacity requirements on each traffic direction. The decomposed three sub-problems from Section 3 are fully discussed in Sections 4, 5, and 6. Based on these, the overall AO algorithm is presented in Section 7. Simulation examples are given in Section 8 to demonstrate the fast convergence and superior performance of the proposed algorithm. Finally, conclusions are given in Section 9.

\section{Power grid topology and signaling paths}

The authors of [8] reported a typical topology of the power grid in a European house as shown in Fig. 1, which enables us to understand how different types of information exchange scheme can be practically implemented under the indoor environment. Under this arrangement, every pair of outlets can be employed as a point-to-point (P2P) communication system, and the corresponding channel is characterized by the wiring topology and load impedance between these two outlets. For example, we choose outlets $T_{1}$ and $T_{2}$ to be the transceiver pair, and refer to them as the terminals in the following. We refer to the shortest cabling link between two terminals as the backbone and its length as the P2P channel's length. Other cables are treated as branches attached to the backbone, which contribute to the multipath nature of the indoor P2P PLC channel. Let us configure the two terminals working in time-division duplex (TDD) mode, i.e., half-duplex constraint is assumed at each terminal. To realize a round of information exchange between terminals $T_{1}$ and $T_{2}$, a two-phase working procedure is needed, where in the first phase terminal $T_{1}$ sends a message via the P2P channel to terminal $T_{2}$, while in the second phase, terminal $T_{2}$ replies a message to terminal $T_{1}$. This procedure has been illustrated in Fig. 2b. We refer to this configuration as the bidirectional direct transmission (BDT) system. Let us denote the transfer function of the P2P channel between $T_{1}$ and $T_{2}$ as $\tilde{H}_{T_{1} T_{2}}(f)$, where $f$ means frequency (Note for simplicity, we drop $f$ in the following). Based on the result 


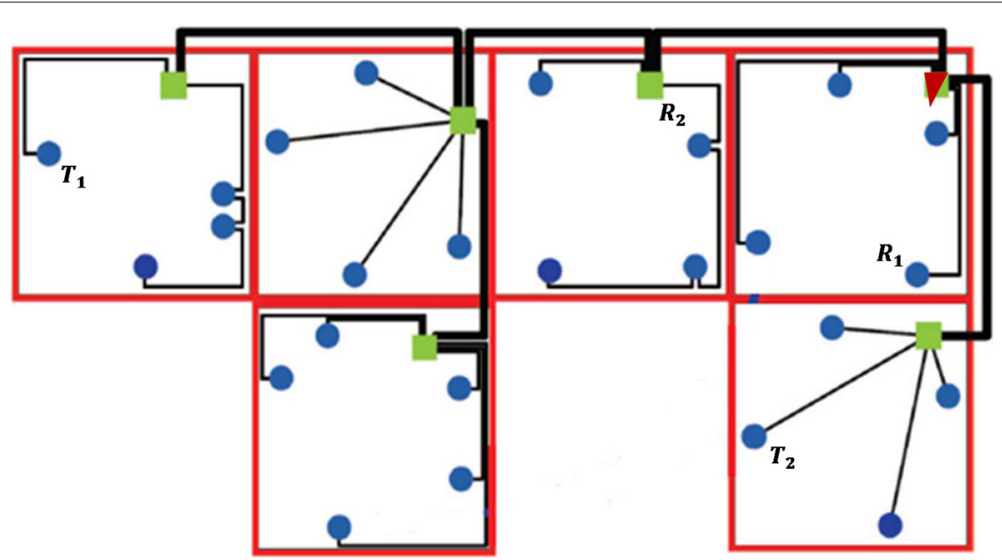

Fig. 1 A typical indoor power grid topology showing main panel (triangle), derivation boxes (squares), and connections with outlets (dots) [8]

in [17] that P2P PLC channels hold a symmetry property, we have

$$
\tilde{H}_{T_{1} T_{2}}=\tilde{H}_{T_{2} T_{1}} \text {. }
$$

From Fig. 1, we find that any outlet located on the backbone of the P2P channel (e.g., $R_{2}$ ) or on a branch of this backbone (e.g., $R_{1}$ ) can be deployed with a plug-in relay device. As the former outlet location can be treated as a special case of the latter one, without losing generality, we choose a branch-located outlet to deploy the relay device and refer to it as the relay node $R$ in the following. After the introduction of the relay node, the original power grid can be seen as consisting of three twoport networks and each of them can be represented by its ABCD matrix, i.e., $\boldsymbol{\Phi}_{T_{1} P}, \boldsymbol{\Phi}_{P R}$, and $\boldsymbol{\Phi}_{T_{2} P}$, respectively. This has been shown in Fig. 3, where point $P$ indicates the intersection of the relay located branch and the backbone. We refer to this situation as the relay-involved PLC channel. Let us denote the signaling path between nodes $L_{1}$ and $L_{2}$ with its complex path gain $H_{L_{1} L_{2}}$, where $L_{1}, L_{2} \in\left\{T_{1}, T_{2}, R\right\}$. It can be analyzed from the transmission line theory that the existence of any branched circuit connection affects the complex channel gain of each signaling path. In general, the transfer function between two terminals changes due to the introduction of relay device, i.e.,

$$
\tilde{H}_{T_{1} T_{2}} \neq H_{T_{1} T_{2}} .
$$

Furthermore, as the terminals and the relay device change their working modes in different signaling stages, even for the same path, its path gain varies over different signaling phases. Note these characteristics are different from the wireless relaying case.

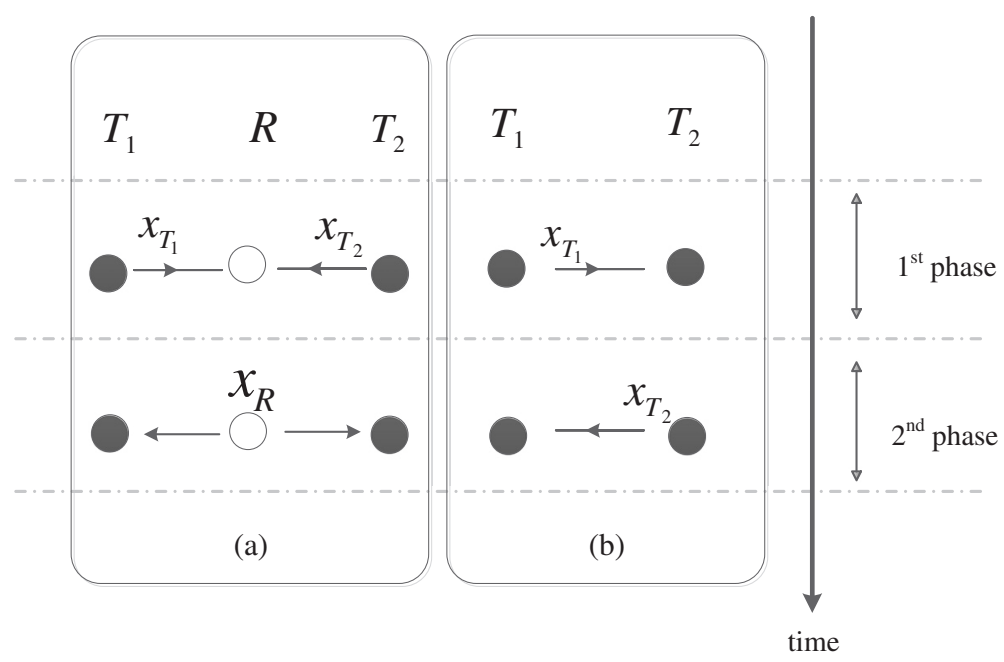

Fig. 2 a R2WX system; b conventional BDT system 


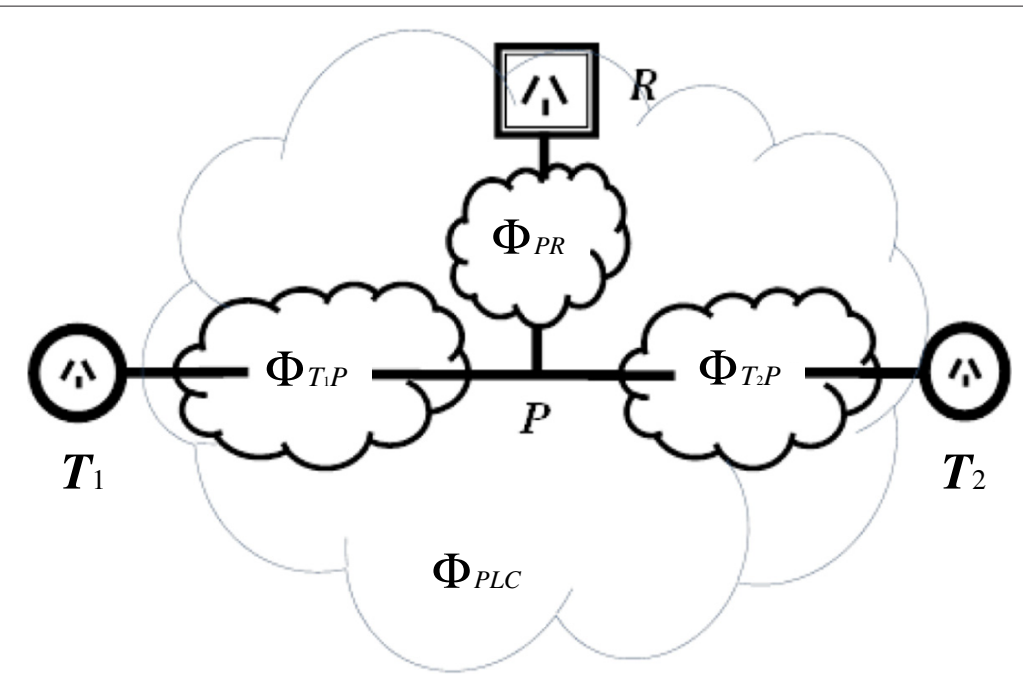

Fig. 3 A relay-involved indoor PLC channel

\section{System model and problem formulation}

The block diagram of the proposed two-way PLC information exchange system is shown in Fig. 4 which consists of three nodes: two information terminals $\left(T_{1}\right.$ and $\left.T_{2}\right)$ which need to exchange data with each other, and a relay node $(R)$ between them. We consider an OFDM-based multicarrier system, where the whole system bandwidth $B$ is uniformly divided into $K$ subcarriers. Given $K$ is a large integer, on each subcarrier, the PLC channel fading can be considered to be frequency-flat. Thus the channel response on the $k$ th $(k=1,2, \ldots, K)$ subcarrier from node $L_{1}$ to node $L_{2}$ is denoted as $h_{L_{1} L_{2}}^{[k],(n)}$, where nodes $L_{1}, L_{2} \in\left\{T_{1}, T_{2}, R\right\}$ and $n=1,2$ is the index of signaling phase.

Let us denote the transmission power on the $k$ th subcarrier from $T_{1}$ as $P_{T_{1}}^{[k]}$, from $T_{2}$ as $P_{T_{2}}^{[k]}$, and from the relay node as $P_{R}^{[k]}$, respectively. Thus, the total network power $P_{\Sigma}$ can be expressed as

$$
P_{\Sigma}=\sum_{k=1}^{K} P_{T_{1}}^{[k]}+\sum_{k=1}^{K} P_{T_{2}}^{[k]}+\sum_{k=1}^{K} P_{R}^{[k]} .
$$

Following the OFDM principle, each packet of information from $T_{1}$ and $T_{2}$ is encoded into $K$ independent complex symbols $X_{1}^{[k]}$ and $X_{2}^{[k]}(k=1,2, \ldots, K)$ of zero mean and unit variance, respectively. To complete two packets of data exchange between two terminals, this twoway relay scheme works in two phases as illustrated in Fig. 2a.

In the first phase, the terminal $T_{1}$ sends information signal $X_{1}^{[k]}(k=1,2, \ldots, K)$ over the $k$ th subcarrier with power $P_{T_{1}}^{[k]}$, at the meantime, the terminal $T_{2}$ sends information signal $X_{2}^{[k]}(k=1,2, \ldots, K)$ over the same subcarrier with power $P_{T_{2}}^{[k]}$. Thus the received signal $Y_{R, 1}^{[k]}$ at the relay node can be written as

$$
Y_{R, 1}^{[k]}=h_{T_{1} R}^{[k],(1)} \sqrt{P_{T_{1}}^{[k]}} X_{1}^{[k]}+h_{T_{2} R}^{[k],(1)} \sqrt{P_{T_{2}}^{[k]}} X_{2}^{[k]}+N_{R, 1}^{[k]}
$$

where $N_{R, 1}^{[k]}$ denotes the $k$ th subchannel noise at the relay node in the first phase.

In the second phase, the relay node amplifies its received sum signal on each sub-carrier with proper amplitude gain $g^{[k]}$, then broadcasts the amplified signal to the terminals $T_{1}$ and $T_{2}$ with power $P_{R}^{[k]}$. Thus the received signals at the two terminals in the second phase are, respectively,

$$
\begin{gathered}
Y_{T_{1}, 2}^{[k]}=h_{R T_{1}}^{[k],(2)} g^{[k]} Y_{R, 1}^{[k]}+N_{T_{1}, 2}^{[k]} \\
Y_{T_{2}, 2}^{[k]}=h_{R T_{2}}^{[k],(2)} g^{[k]} Y_{R, 1}^{[k]}+N_{T_{2}, 2}^{[k]}
\end{gathered}
$$

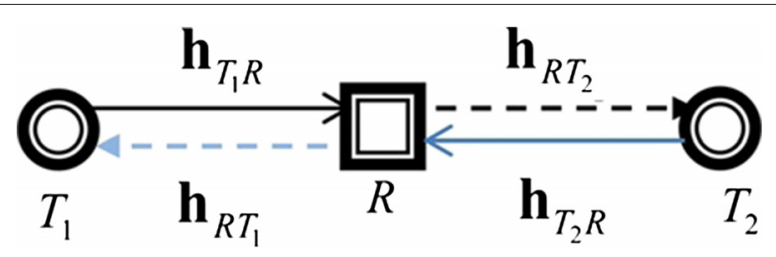

Fig. 4 A multicarrier two-way relay system where the solid lines and dash lines indicate phase 1 and 2 , respectively. $T_{1}, T_{2}$, and $R$ stand for the first terminal, the second terminal, and the relay node, respectively 
where $N_{T_{1}, 2}^{[k]}$ and $N_{T_{2}, 2}^{[k]}$ denote the $k$ th subchannel noise at the terminal $T_{1}$ and $T_{2}$, respectively, in the second phase, and

$$
g^{[k]}=\sqrt{\frac{P_{R}^{[k]}}{P_{T_{1}}^{[k]}\left|h_{T_{1} R}^{[k],(1)}\right|^{2}+P_{T_{2}}^{[k]}\left|h_{T_{2} R}^{[k],(1)}\right|^{2}+W_{R}^{[k]}} .}
$$

Let us assume the power spectral density (PSD) of noise does not change in two successive phases, and use $W_{L_{2}}^{[k]}$ in (7) to denote the power of noise $N_{L_{2}, n}^{[k]}(n=1,2)$ at node $L_{2}$. Substituting (4) and (7) into (5) and (6) leads to

$$
\begin{aligned}
& Y_{T_{1}, 2}^{[k]}=h_{T_{1} R}^{[k],(1)} h_{R T_{1}}^{[k],(2)} \sqrt{\frac{P_{R}^{[k]} P_{T_{1}}^{[k]}}{P_{T_{1}}^{[k]}\left|h_{T_{1} R}^{[k],(1)}\right|^{2}+P_{T_{2}}^{[k]}\left|h_{T_{2} R}^{[k],(1)}\right|^{2}+W_{R}^{[k]}}} X_{1}^{[k]} \\
& +h_{T_{2} R}^{[k],(1)} h_{R T_{1}}^{[k],(2)} \sqrt{\frac{P_{R}^{[k]} P_{T_{2}}^{[k]}}{P_{T_{1}}^{[k]}\left|h_{T_{1} R}^{[k],(1)}\right|^{2}+P_{T_{2}}^{[k]}\left|h_{T_{2} R}^{[k],(1)}\right|^{2}+W_{R}^{[k]}}} X_{2}^{[k]} \\
& +h_{R T_{1}}^{[k],(2)} \sqrt{\frac{P_{R}^{[k]}}{P_{T_{1}}^{[k]}\left|h_{T_{1} R}^{[k],(1)}\right|^{2}+P_{T_{2}}^{[k]}\left|h_{T_{2} R}^{[k],(1)}\right|^{2}+W_{R}^{[k]}}} N_{R, 1}^{[k]}+N_{T_{1}, 2}^{[k]} \\
& Y_{T_{2}, 2}^{[k]}=h_{T_{2} R}^{[k],(1)} h_{R T_{2}}^{[k],(2)} \sqrt{\frac{P_{R}^{[k]} P_{T_{2}}^{[k]}}{P_{T_{1}}^{[k]}\left|h_{T_{1} R}^{[k],(1)}\right|^{2}+P_{T_{2}}^{[k]}\left|h_{T_{2} R}^{[k],(1)}\right|^{2}+W_{R}^{[k]}}} X_{2}^{[k]} \\
& +h_{T_{1} R}^{[k],(1)} h_{R T_{2}}^{[k],(2)} \sqrt{\frac{P_{R}^{[k]} P_{T_{1}}^{[k]}}{P_{T_{1}}^{[k]}\left|h_{T_{1} R}^{[k],(1)}\right|^{2}+P_{T_{2}}^{[k]}\left|h_{T_{2} R}^{[k],(1)}\right|^{2}+W_{R}^{[k]}}} X_{1}^{[k]} \\
& +h_{R T_{2}}^{[k],(2)} \sqrt{\frac{P_{R}^{[k]}}{P_{T_{1}}^{[k]}\left|h_{T_{1} R}^{[k],(1)}\right|^{2}+P_{T_{2}}^{[k]}\left|h_{T_{2} R}^{[k],(1)}\right|^{2}+W_{R}^{[k]}}} N_{R, 1}^{[k]}+N_{T_{2}, 2}^{[k]} .
\end{aligned}
$$

We assume that all the channel state information (CSI) is known by the two terminals. Furthermore, as $T_{1}$ has full knowledge of $X_{1}^{[k]}$, the first term in (8) can be readily eliminated and the remainder is used to decode the information beard by signal $X_{2}^{[k]}$. Similar process is applied in (9). Let us denote the SNR of the $k$ th subcarrier at terminal $T_{1}$ as $\mathrm{SNR}_{T_{1}}^{[k]}$, and terminal $T_{2}$ as $\mathrm{SNR}_{T_{2}}^{[k]}$, and we can calculate

$$
\begin{aligned}
\mathrm{SNR}_{T_{1}}^{[k]} & =\frac{P_{R}^{[k]} \gamma_{T_{2} R}^{[k],(1)} P_{T_{2}}^{[k]} \gamma_{R T_{1}}^{[k],(2)}}{1+P_{R}^{[k]} \gamma_{R T_{1}}^{[k],(2)}+P_{T_{1}}^{[k]} \gamma_{T_{1} R}^{[k],(1)}+P_{T_{2}}^{[k]} \gamma_{T_{2} R}^{[k],(1)}} \\
\mathrm{SNR}_{T_{2}}^{[k]} & =\frac{P_{R}^{[k]} \gamma_{T_{1} R}^{[k],(1)} P_{T_{1}}^{[k]} \gamma_{R T_{2}}^{[k],(2)}}{1+P_{R}^{[k]} \gamma_{R T_{2}}^{[k],(2)}+P_{T_{1}}^{[k]} \gamma_{T_{1} R}^{[k],(1)}+P_{T_{2}}^{[k]} \gamma_{T_{2} R}^{[k],(1)}}
\end{aligned}
$$

where the normalized path gain $\gamma_{L_{1} L_{2}}^{[k],(n)}$ of subchannel $h_{L_{1} L_{2}}^{[k],(n)}$ is defined as

$$
\gamma_{L_{1} L_{2}}^{[k],(n)}=\frac{\left|h_{L_{1} L_{2}}^{[k](n)}\right|^{2}}{W_{L_{2}}^{[k]}}
$$

Thus, we can obtain the averaged sub-channel capacity (ASC) of the data traffic on the direction from $T_{2}$ to $T_{1}$ as

$$
\bar{C}_{1}=\frac{1}{2 K} \sum_{k=1}^{K} \log _{2}\left(1+\frac{P_{R}^{[k]} \gamma_{T_{2} R}^{[k],(1)} P_{T_{2}}^{[k]} \gamma_{R T_{1}}^{[k],(2)}}{1+P_{R}^{[k]} \gamma_{R T_{1}}^{[k],(2)}+P_{T_{1}}^{[k]} \gamma_{T_{1} R}^{[k],(1)}+P_{T_{2}}^{[k]} \gamma_{T_{2} R}^{[k],(1)}}\right)
$$

where $\bar{C}_{1}$ is measured in bits/s/Hz/subcarrier, and the factor $\frac{1}{2}$ indicate the two-phase characteristic of the system configuration. Similarly, we can express the ASC of the data-link on the reverse traffic direction, namely from $T_{1}$ to $T_{2}$, as

$$
\bar{C}_{2}=\frac{1}{2 K} \sum_{k=1}^{K} \log _{2}\left(1+\frac{P_{R}^{[k]} \gamma_{T_{1} R}^{[k],(1)} P_{T_{1}}^{[k]} \gamma_{R T_{2}}^{[k],(2)}}{1+P_{R}^{[k]} \gamma_{R T_{2}}^{[k],(2)}+P_{T_{1}}^{[k]} \gamma_{T_{1} R}^{[k],(1)}+P_{T_{2}}^{[k]} \gamma_{T_{2} R}^{[k],(1)}}\right) .
$$

With the QoS criteria as the lower-bounds of the ASC on two traffic directions, to make the most efficient utilization of the system total power we propose the following optimization problem

$$
\begin{aligned}
\min _{P_{T_{1}}^{[k]}, P_{T_{2}}^{[k]}, P_{R}^{[k]}} & P_{\Sigma} \\
\text { s.t. } & \bar{C}_{1} \geq \bar{q}_{1}, \\
& \bar{C}_{2} \geq \bar{q}_{2}, \\
& P_{T_{1}}^{[k]}, P_{T_{2}}^{[k]}, P_{R}^{[k]} \geq 0, \forall k
\end{aligned}
$$

where (15) is the objective function of the total network transmission power, and $\bar{q}_{1}, \bar{q}_{2} \geq 0$ are the required minimal ASC on each traffic direction to support certain applications.

Note the problem formulated in (15)-(18) has twofold tasks. On the one hand, it should distribute the system total power optimally on each subcarrier. On the other hand, the power of each subcarrier needs to be optimally allocated to the two terminal nodes and the relay node. As the relay-involved PLC channel is naturally time-varying as we mentioned in Section 2, this problem cannot be solved by the two-layered approach used in [18].

We mention that in the problem (15)-(18), $\bar{q}_{1}$ does not have to be equal to $\bar{q}_{2}$. For example, most of the time in PLC networks, the down-link QoS is more critical than the up-link one with regard to the user experience. For some scenarios where the QoS requirements do not need to be specified on each individual traffic direction, we can also define the system information exchange capacity (in bits/s/Hz) as

$$
C_{\epsilon}=K\left(\bar{C}_{1}+\bar{C}_{2}\right) .
$$


So that from (16) and (17) we can write

$$
C_{\epsilon} \geq q_{\epsilon}
$$

where the minimal data rate $q_{\epsilon}=K\left(\bar{q}_{1}+\bar{q}_{2}\right)$. Note (20) is the necessary, but not sufficient condition for (16) and (17), thus the problem

$$
\begin{aligned}
\min _{P_{T_{1}}^{[k]}, P_{T_{2}}^{[k]}, P_{R}^{[k]}} & P_{\Sigma} \\
\text { s.t. } & C_{\epsilon} \geq q_{\epsilon}, \\
& P_{T_{1}}^{[k]}, P_{T_{2}}^{[k]}, P_{R}^{[k]} \geq 0, \forall k
\end{aligned}
$$

is a special case of the problem (15)-(18).

The exact solution to the problem (15)-(18) is difficult to obtain because the system minimal ASC constraints in (16) and (17) are non-convex. In this paper, we provide a local optimum solution by adopting the AO approach from [16], where we first optimize $P_{R}^{[k]}$ with previously given $P_{T_{1}}^{[k]}$ and $P_{T_{2}}^{[k]}, k=1,2, \ldots, K$; then optimize $P_{T_{1}}^{[k]}$ with previously optimized $P_{R}^{[k]}$ and given $P_{T_{2}}^{[k]}$; next we optimize $P_{T_{2}}^{[k]}$ with previously optimized $P_{R}^{[k]}$ and $P_{T_{1}}^{[k]}$. Repeat this process until convergence, i.e., the difference between the total system power $P_{\Sigma}$ obtained in two consecutive iterations is less than a preset threshold. For any two groups of fixed power allocation parameters, the resulted subproblem becomes convex or quasi-convex. Based on this method, we develop the overall algorithm to solve the original problem (15)-(18). We discuss these issues in detail from Section 4 to Section 6.

\section{Optimal relay power allocation with given terminal power allocations}

Let $x_{k}$ stand for $P_{R}^{[k]}(k=1,2, \ldots, K)$. For fixed $P_{T_{1}}^{[k]}$ and $P_{T_{2}}^{[k]}$, the problem (15)-(18) becomes

$$
\begin{array}{ll}
\min _{x_{k}} & \sum_{k=1}^{K} x_{k} \\
\text { s.t. } & 2 K \bar{q}_{1}-\sum_{k=1}^{K} \log _{2}\left(A_{k}-\frac{B_{k}}{C_{k} x_{k}+D_{k}}\right) \leq 0 \\
& 2 K \bar{q}_{2}-\sum_{k=1}^{K} \log _{2}\left(E_{k}-\frac{F_{k}}{G_{k} x_{k}+D_{k}}\right) \leq 0 \\
& x_{k} \geq 0, \forall k
\end{array}
$$

where

$$
\begin{aligned}
& A_{k}=1+P_{T_{2}}^{[k]} \gamma_{T_{2} R}^{[k],(1)} \\
& B_{k}=P_{T_{2}}^{[k]} \gamma_{T_{2} R}^{[k],(1)}\left(1+P_{T_{1}}^{[k]} \gamma_{T_{1} R}^{[k],(1)}+P_{T_{2}}^{[k]} \gamma_{T_{2} R}^{[k],(1)}\right) \\
& C_{k}=\gamma_{R T_{1}}^{[k],(2)} \\
& D_{k}=1+P_{T_{1}}^{[k]} \gamma_{T_{1} R}^{[k],(1)}+P_{T_{2}}^{[k]} \gamma_{T_{2} R}^{[k],(1)} \\
& E_{k}=1+P_{T_{1}}^{[k]} \gamma_{T_{1} R}^{[k],(1)} \\
& F_{k}=P_{T_{1}}^{[k]} \gamma_{T_{1} R}^{[k],(1)}\left(1+P_{T_{1}}^{[k]} \gamma_{T_{1} R}^{[k],(1)}+P_{T_{2}}^{[k]} \gamma_{T_{2} R}^{[k],(1)}\right) \\
& G_{k}=\gamma_{R T_{2}}^{[k],(2)} .
\end{aligned}
$$

Let us write down the Karush-Kuhn-Tucker (KKT) conditions [19] to the problem (24)-(27) as, for $\forall k$,

$$
\begin{aligned}
1-\lambda_{1} \frac{B_{k} C_{k}}{\ln 2\left(A_{k}-\frac{B_{k}}{C_{k} x_{k}+D_{k}}\right)\left(C_{k} x_{k}+D_{k}\right)^{2}} & =0 \\
-\lambda_{2} \frac{F_{k} G_{k}}{\ln 2\left(E_{k}-\frac{F_{k}}{G_{k} x_{k}+D_{k}}\right)\left(G_{k} x_{k}+D_{k}\right)^{2}} & =0 \\
\lambda_{1}\left[2 K \bar{q}_{1}-\sum_{k=1}^{K} \log _{2}\left(A_{k}-\frac{B_{k}}{C_{k} x_{k}+D_{k}}\right)\right] & =0 \\
\lambda_{2}\left[2 K \bar{q}_{2}-\sum_{k=1}^{K} \log _{2}\left(E_{k}-\frac{F_{k}}{G_{k} x_{k}+D_{k}}\right)\right] & =0 \\
\lambda_{1}, \lambda_{2} & \geq 0 \\
x_{k} & \geq 0 .
\end{aligned}
$$

Proposition 1. The problem (24)-(27) is convex on $\left\{x_{k} \mid x_{k} \geq 0, k=1,2, \ldots, K\right\}$. For fixed $\lambda_{1}, \lambda_{2}>0$, the lefthand-side (LHS) of (35)-(37) are all monotone functions of $x_{k}$.

\section{Proof. See Appendix 1.}

Form Proposition 1, a bisection search algorithm can be employed to solve the KKT conditions (35)-(39), which leads to the optimal solution of the problem (24)-(27).

\section{Optimal first terminal power allocation with given second terminal and relay power allocations}

Let $y_{k}$ stand for $P_{T_{1}}^{[k]}(k=1,2, \ldots, K)$, then for given $P_{R}^{[k]}$ and $P_{T_{2}}^{[k]}$, we now consider the optimal power allocation of the first terminal by solving the problem of 


$$
\begin{array}{ll}
\min _{y_{k}} & \sum_{k=1}^{K} y_{k} \\
\text { s.t. } & 2 K \bar{q}_{1}-\sum_{k=1}^{K} \log _{2}\left(1+\frac{H_{k}}{J_{k} y_{k}+L_{k}}\right) \leq 0 \\
& 2 K \bar{q}_{2}-\sum_{k=1}^{K} \log _{2}\left(M_{k}-\frac{O_{k}}{J_{k} y_{k}+P_{k}}\right) \leq 0 \\
& y_{k} \geq 0, \forall k
\end{array}
$$

where

$$
\begin{aligned}
H_{k} & =P_{R}^{[k]} \gamma_{T_{2} R}^{[k],(1)} P_{T_{2}}^{[k]} \gamma_{R T_{1}}^{[k],(2)} \\
J_{k} & =\gamma_{T_{1} R}^{[k],(1)} \\
L_{k} & =1+P_{R}^{[k]} \gamma_{R T_{1}}^{[k],(2)}+P_{T_{2}}^{[k]} \gamma_{T_{2} R}^{[k],(1)} \\
M_{k} & =1+P_{R}^{[k]} \gamma_{R T_{2}}^{[k],(2)} \\
O_{k} & =P_{R}^{[k]} \gamma_{R T_{2}}^{[k],(2)}\left(1+P_{R}^{[k]} \gamma_{R T_{2}}^{[k],(2)}+P_{T_{2}}^{[k]} \gamma_{T_{2} R}^{[k],(1)}\right) \\
P_{k} & =1+P_{R}^{[k]} \gamma_{R T_{2}}^{[k],(2)}+P_{T_{2}}^{[k]} \gamma_{T_{2} R}^{[k],(1)} .
\end{aligned}
$$

Let us write down the KKT conditions to the problem (40)-(43) as, for $\forall k$,

$$
\begin{aligned}
1-\mu_{1} \frac{H_{k} J_{k}}{\ln 2\left(-1-\frac{H_{k}}{J_{k} y_{k}+L_{k}}\right)\left(J_{k} y_{k}+L_{k}\right)^{2}} & =0 \\
-\mu_{2} \frac{J_{k} O_{k}}{\ln 2\left(M_{k}-\frac{O_{k}}{J_{k} y_{k}+P_{k}}\right)\left(J_{k} y_{k}+P_{k}\right)^{2}} & =0 \\
\mu_{1}\left[2 K \bar{q}_{1}-\sum_{k=1}^{K} \log _{2}\left(1+\frac{H_{k}}{J_{k} y_{k}+L_{k}}\right)\right] & =0 \\
\mu_{2}\left[2 K \bar{q}_{2}-\sum_{k=1}^{K} \log _{2}\left(M_{k}-\frac{O_{k}}{J_{k} y_{k}+P_{k}}\right)\right] & =0 \\
\mu_{1}, \mu_{2} & \geq 0 \\
y_{k} & \geq 0 .
\end{aligned}
$$

Proposition 2. The problem (40)-(43) is quasi-convex on $\left\{y_{k} \mid y_{k} \geq 0, k=1,2, \ldots, K\right\}$. And for fixed $\mu_{1}, \mu_{2}>0$, the LHS of -(52) are monotone functions of $y_{k}$.

Proof. See Appendix 2.

Based on Proposition 2, the solution to the problem (40)-(43) can be obtained by using a bisection search algorithm.

\section{Optimal second terminal power allocation with given first terminal and relay power allocations}

Similarly, based on the optimized $P_{T_{1}}^{[k]}$ and $P_{R}^{[k]}$ from above, we now denote $P_{T_{2}}^{[k]}$ as $z_{k}$ and consider the optimization of the second terminal's power allocation by solving the problem of

$$
\begin{array}{ll}
\min _{z_{k}} & \sum_{k=1}^{K} z_{k} \\
\text { s.t. } & 2 K \bar{q}_{1}-\sum_{k=1}^{K} \log _{2}\left(Q_{k}-\frac{R_{k}}{S_{k} z_{k}+T_{k}}\right) \leq 0 \\
& 2 K \bar{q}_{2}-\sum_{k=1}^{K} \log _{2}\left(1+\frac{U_{k}}{S_{k} z_{k}+V_{k}}\right) \leq 0 \\
& z_{k} \geq 0, \forall k
\end{array}
$$

where

$$
\begin{aligned}
Q_{k} & =1+P_{R}^{[k]} \gamma_{R T_{1}}^{[k],(2)} \\
R_{k} & =P_{R}^{[k]} \gamma_{R T_{1}}^{[k],(2)}\left(1+P_{R}^{[k]} \gamma_{R T_{1}}^{[k],(2)}+P_{T_{1}}^{[k]} \gamma_{T_{1} R}^{[k],(1)}\right) \\
S_{k} & =\gamma_{T_{2} R}^{[k],(1)} \\
T_{k} & =1+P_{R}^{[k]} \gamma_{R T_{1}}^{[k],(2)}+P_{T_{1}}^{[k]} \gamma_{T_{1} R}^{[k],(1)} \\
U_{k} & =P_{R}^{[k]} \gamma_{T_{1} R}^{[k],(1)} P_{T_{1}}^{[k]} \gamma_{R T_{2}}^{[k],(2)} \\
V_{k} & =1+P_{R}^{[k]} \gamma_{R T_{2}}^{[k],(2)}+P_{T_{1}}^{[k]} \gamma_{T_{1} R}^{[k],(1)}
\end{aligned}
$$
$\forall k$

The KKT conditions to the problem (55)-(58) are, for

$$
\begin{aligned}
1-\delta_{1} \frac{R_{k} S_{k}}{\ln 2\left(Q_{k}-\frac{R_{k}}{S_{k} z_{k}+T_{k}}\right)\left(S_{k} z_{k}+T_{k}\right)^{2}} & =0 \\
-\delta_{2} \frac{S_{k} U_{k}}{\ln 2\left(-1-\frac{U_{k}}{S_{k} z_{k}+V_{k}}\right)\left(S_{k} z_{k}+V_{k}\right)^{2}} & =0 \\
\delta_{1}\left[2 K \bar{q}_{1}-\sum_{k=1}^{K} \log _{2}\left(Q_{k}-\frac{R_{k}}{S_{k} z_{k}+T_{k}}\right)\right] & =0 \\
\delta_{2}\left[2 K \bar{q}_{2}-\sum_{k=1}^{K} \log _{2}\left(1+\frac{U_{k}}{S_{k} z_{k}+V_{k}}\right)\right] & =0 \\
\delta_{1}, \delta_{2} & \geq 0 \\
z_{k} & \geq 0 .
\end{aligned}
$$

Proposition 3. The problem (55)-(58) is quasi-convex on $\left\{z_{k} \mid z_{k} \geq 0, k=1,2, \ldots, K\right\}$. And for fixed $\delta_{1}, \delta_{2}>0$, the LHS of (65)-(67) are monotonically changing with $z_{k}$.

Note the structural similarity between the problem (40)-(43) and the problem (55)-(58). The proof of Proposition 3 is similar to the proof of Proposition 2 in Appendix 2.

Again, the solution to the problem (40)-(43) can be obtained by using a conventional bisection algorithm. 


\section{Overall iterative algorithm and implementation issues}

Based on the discussion in Sections 4, 5 and 6, we summarize the proposed $\mathrm{AO}$ algorithm for solving the problem (15)-(18). This is shown in Table 1.

\subsection{Converge of the $\mathrm{AO}$ algorithm}

In general, the alternating optimization method cannot guarantee to converge to the globally optimal solution. However, since we obtain the global optimum for each sub-problem, the proposed $\mathrm{AO}$ algorithm converges to a stationary point of the objective function (15). This conclusion has been proved in Appendix 3 and verified by simulation examples in Section 8.

\subsection{Complexity analysis}

The computational complexity of the proposed AO Algorithm can be analyzed as follows. In Table 1, the second step is used to solve the first sub-problem (35)-(39). For fixed $\lambda_{1}, \lambda_{2}$, and $x_{k}, 18$ real number multiplications are needed to calculate the LHS of (35). Thus, the complexity order of solving the sub-problem (35)(39) is $\mathcal{O}\left(18 K c_{x} c_{\lambda}\right)$, where $c_{x}$ is the number of bi-section searches to obtain $x_{k}$, and $c_{\lambda}$ is the number of iterations to obtain $\lambda_{1}$ and $\lambda_{2}$. Similarly, the third step and fourth step in Algorithm 1 require a complex order of $\mathcal{O}\left(18 K c_{y} c_{\mu}\right)$ and $\mathcal{O}\left(18 K c_{z} c_{\delta}\right)$, respectively, where $c_{y}, c_{\mu}, c_{z}$, and $c_{\delta}$ are the number of iterations required to obtain $y_{k},\left(\mu_{1}, \mu_{2}\right)$, $z_{k}$, and $\left(\delta_{1}, \delta_{2}\right)$, respectively.

Therefore, the per-iteration computational complexity order of the proposed $\mathrm{AO}$ algorithm is $\mathcal{O}\left(18 K\left(c_{x} c_{\lambda}+\right.\right.$ $\left.c_{y} c_{\mu}+c_{z} c_{\delta}\right)$ ), which increases linearly with the number of subcarriers. The number of iterations of the $\mathrm{AO}$ algorithm till convergence depends on the stop criterion $\sigma$. It will

Table 1 AO algorithm for solving the problem (15)-(18)

1) Initialize $P_{T_{1}}^{[1]}=p_{t 1}$ while $P_{T_{1}}^{[k]}=0(k=2,3, \ldots, K) ; P_{T_{2}}^{[1]}=p_{t 2}$ while
$P_{T_{2}}^{[k]}=0(k=2,3, \ldots, K) ;$ and $P_{R}^{[1]}=p_{r}$ while $P_{R}^{[k]}=0(k=2,3, \ldots, K)$.
Here the scalars $p_{t 1}, p_{t 2}$ and $p_{r}$ are positive numbers which satisfy (16)
and (17).
2) Use bi-section algorithm with preselected (newly obtained) $P_{T_{1}}^{[k]}$
and $P_{T_{2}}^{[k]}$ to solve (35)-(39) to find a new set of $P_{R}^{[k]}$.
3) Use bi-section algorithm with the preselected (newly obtained) $P_{T_{2}}^{[k]}$ and
$P_{R}^{[k]}$ to solve (50)-(54) to find a new set of $P_{T_{1}}^{[k]}$.
4) Use bi-section algorithm with newly obtained $P_{R}^{[k]}$ and $P_{T_{1}}^{[k]}$ to solve
(65)-(69) to find a new set of $P_{T_{2}}^{[k]}$.
5) Calculate the total power value (3) with the obtained power allocation
values from steps 2-4.
6) If the difference of the value of (3) in two successive iterations is less
than a preset threshold $\sigma$, then stop; otherwise, go to step 2.

be shown in the next section that about six iterations are required to achieve $\sigma=10^{-5}$.

\subsection{Implementation issues}

Based on [20] which reported that indoor PLC channels have relatively long coherent time, we can reasonably treat $h_{L_{1} L 2}^{[k],(n)}\left(n=1,2, k=1, \cdots, K, L_{1}, L_{2} \in\left\{T_{1}, T_{2}, R\right\}\right)$ as quasi-static. In practice, the CSI of $h_{T_{1} R}^{[k],(1)}$ and $h_{T_{2} R}^{[k],(1)}$ can be obtained at the relay node through standard channel training method in the first signaling phase, and then forwarded to two terminals in the second phase. Similarly, in the second phase, terminal $T_{j}, j=1,2$, can obtain the CSI of $h_{R T_{j}}^{[k],(2)}$ via channel training. Then, the second-phase $\operatorname{CSI} h_{R T_{j}}^{[k],(2)}$ is fed back to the relay node so that the power allocation for each node can be calculated (through the proposed AO algorithm) at the relay node and the results are sent to each terminal.

\section{Numerical examples}

In this section, we present simulation results based on a simple testing relay-involved PLC channel. Its topology is given in Fig. 5, where different types of cables have been used for different segments of the network. The parameters for each type of cable can be found in [20]. Moreover, the physical (PHY) layer specification of the HomePlug AV2 protocol [21] has been applied to set the simulation parameters.

(1) The noise of indoor PLC system can be modeled as colored Gaussian noise [22], and the PLC noise model in [23] has been adopted to generate the testing noise. For simplicity, we assume that $N_{R, 1}^{[k]}, N_{T_{1}, 2}^{[k]}$, and $N_{T_{2}, 2}^{[k]}$ are independent and identically distributed (i.i.d.) and share the PSD as shown in Fig. 6 , where $k=1,2, \ldots, 1155$ is the subcarrier index.

(2) In the proposed system, all three nodes work in the TDD mode, i.e., each of them acts either as a transmitter $(\mathrm{Tx})$ or receiver $(\mathrm{Rx})$ in different signaling phase. They can be modeled by the common circuit structure as shown in Fig. 7. For simplicity, we assume that all three nodes share the same parameters, i.e., in the Tx mode, they have an inner impedance $Z_{g}=50 \Omega$, while in the $\mathrm{Rx}$ mode, they have a loading impedance $Z_{l}=150 \Omega$.

(3) A preexisting load $Z_{0}$ (which can represent an appliance, such as a dishwasher) has been deployed on the outlet where the relay device is plugged in. The frequency response of $Z_{0}$ is given in Fig. 8.

(4) With the settings shown above, each signaling path in the relay-involved channel can be treated as a P2P channel, and the latter one can be easily realized by using some published P2P PLC channel models, e.g., here we adopt the model presented in [20]. Thus a group of correlated 


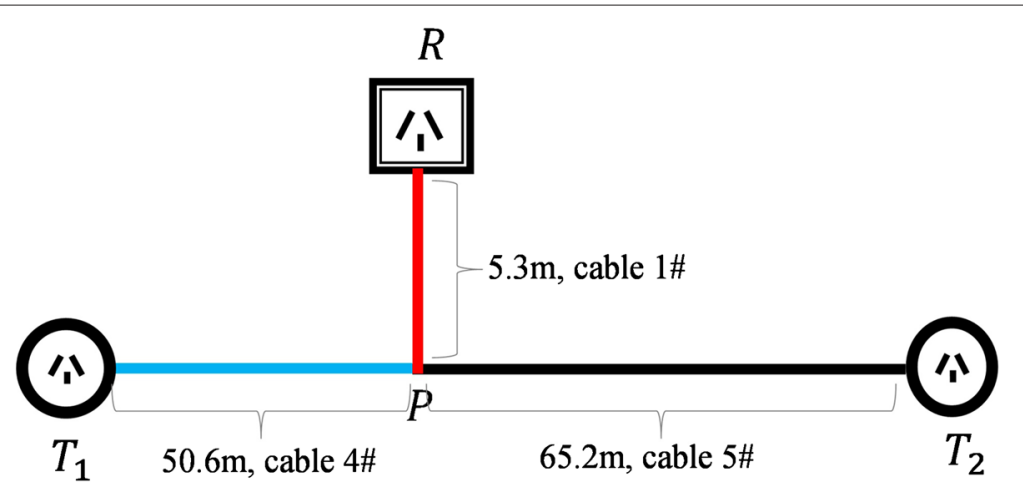

Fig. 5 A testing power grid topology

path gains $\left(h_{T_{1} R}^{[k],(1)}, h_{T_{2} R}^{[k],(1)}, h_{R T_{1}}^{[k],(2)}\right.$, and $\left.h_{R T_{2}}^{[k],(2)}\right)$ can be generated as shown in Fig. 9. Note in Fig. 9 the P2P channel gain $\tilde{h}_{T_{1} T_{2}}^{[k]}$ has also been given, which corresponds to the situation where the relay device is unplugged from the grid.

(5) Using (12), the normalized path gains $\left(\gamma_{T_{1} R}^{[k],(1)}\right.$, $\gamma_{T_{2} R}^{[k],(1)}, \gamma_{R T_{1}}^{[k],(2)}$, and $\left.\gamma_{R T_{2}}^{[k],(2)}\right)$ on each subcarrier can be calculated and they are shown in Fig. 10.

The parameters used for simulation are summarized in Table 2. In addition, for the proposed iterative algorithm, we set the convergence condition as the difference between the total power obtained in two consecutive iterations less than $10^{-5}$.

As an example to demonstrate the convergence speed of the proposed AO algorithm, Fig. 11 shows the total transmission power versus the number of iterations when directional ASC $\bar{q}_{1}$ in (16) is fixed as 2 bits/s/Hz/subcarrier, while $\bar{q}_{2}$ in (17) is set to five different values from 1 to $3 \mathrm{bits} / \mathrm{s} / \mathrm{Hz} / \mathrm{subcarrier}$, respectively. It can be seen that the proposed $\mathrm{AO}$ algorithm converges typically within ten iterations. In particular, the decreasing of the total power after six iterations is very small. Thus only a few iterations are required to achieve a good performance. This also indicates that the $\mathrm{AO}$ algorithm has a short processing delay, which is important for practical relay-assisted PLC systems. It can also be observed from Fig. 11 that with the increasing of one directional ASC $\bar{q}_{2}$, more transmission power is needed to meet the stricter QoS constraint on this traffic direction, which reflects the typical QoS-cost trade-off in communication systems.

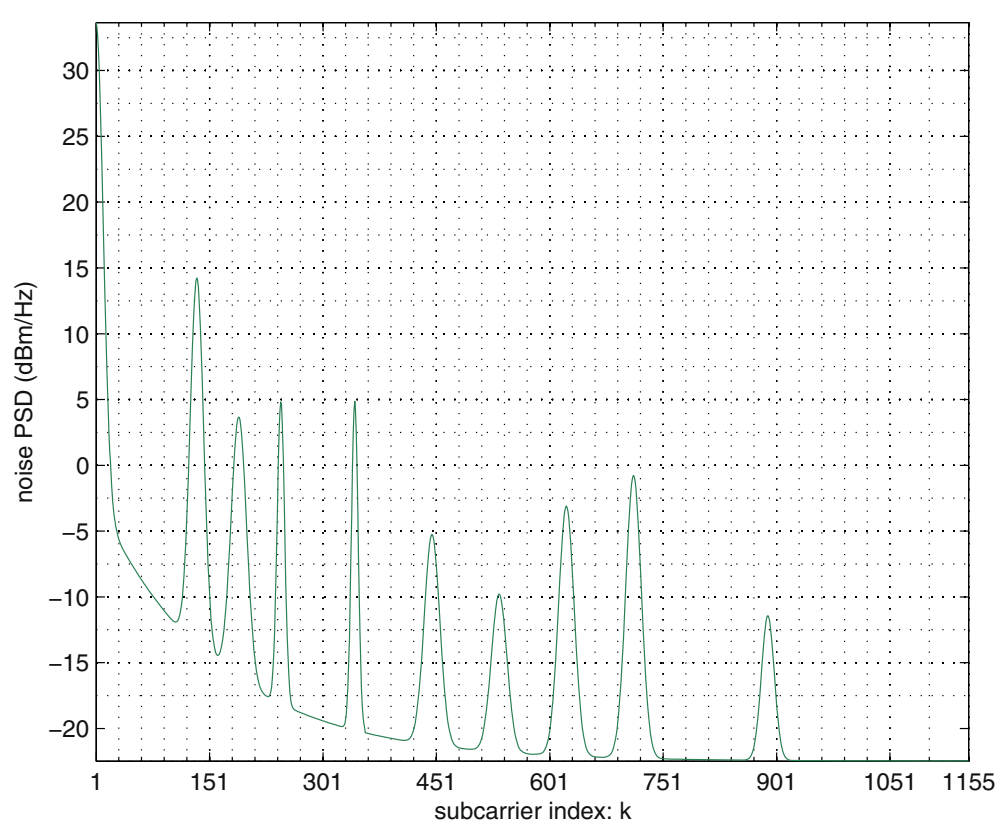

Fig. 6 Common testing noise PSD on every node of the PLC network 


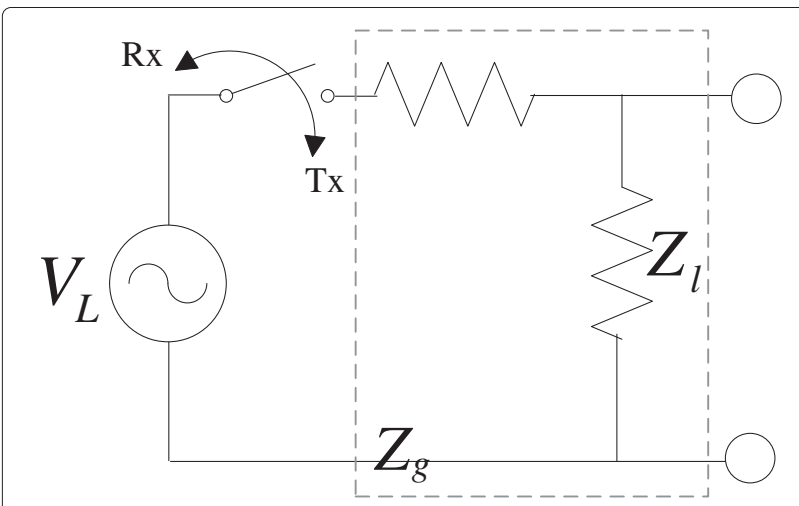

Fig. 7 Common circuit structure to model the node $L$ working in $T x$ or Rx mode where $L \in\left\{T_{1}, T_{2}, R\right\}$

Next, under the same testing PLC system, we compare the proposed R2WX scheme (which is optimized by the AO power allocation algorithm) with the BDT system. To ensure a fair comparison, we configure the BDT system working as follows. In the first phase, terminal $T_{1}$ sends information signal $X_{1}^{[k]}(k=1,2, \ldots, K)$ with power $D_{T_{1}}^{[k]}$ over the $k$ th subcarrier to terminal $T_{2}$ subjecting to the directional QoS requirement (17). As reported in [15] that to meet the ASC constraint on this traffic direction, the optimal power allocation at $T_{1}$ is the classical water-filling algorithm. In the second phase, the terminal $T_{2}$ replies information signal $X_{2}^{[k]}(k=1,2, \ldots, K)$ with power $D_{T_{2}}^{[k]}$ to terminal $T_{1}$ subjecting to the QoS requirement on this traffic direction (16). Again the water-filling algorithm is used for the power allocation at $T_{2}$. Thus, the total network power of the BDT system is calculated as

$$
P_{\mathrm{BDT}}=\sum_{k=1}^{K} D_{T_{1}}^{[k]}+\sum_{k=1}^{K} D_{T_{2}}^{[k]}
$$

The total network power versus the common QoS requirements curve for the BDT system and R2WX system is shown in Fig. 12, where we fix $\bar{q}_{2}$ at $1.5 \mathrm{bits} / \mathrm{s} / \mathrm{Hz} / \mathrm{subcarrier}$, and increase $\bar{q}_{1}$ from 1 to 4 bits/s/Hz/subcarrier. We can see from Fig. 12 that with respect to the $\mathrm{BDT}$ system, the $\mathrm{AO}$ algorithm makes the R2WX system meet the QoS requirements with much less power consumption.

As an example to show the power allocation on each subcarrier for the BDT and R2WX systems, powers on 70 subchannels (from 631 to 700 ) are shown in Figs. 13 and 14, respectively, where the ASC on two traffic directions $\left(\bar{q}_{1}\right.$ and $\left.\bar{q}_{2}\right)$ are set to 2.5 and $1.5 \mathrm{bits} / \mathrm{s} / \mathrm{Hz} / \mathrm{subcarrier}$, respectively. In Fig. 13, we can see that the terminal $T_{2}$ spends more power than $T_{1}$ on each subchannel, which is a result of the stricter QoS requirement $\bar{q}_{1}$ on this traffic direction. Despite this, the power allocations in both terminals follow a similar pattern, which reflects the characteristics of the water-filling algorithm. On the other hand, the power allocation for the R2WX system shown in Fig. 14 demonstrates that the AO algorithm not only considers the direct path's characteristics but also takes the property of the terminal-to-relay links into account. This leads to a more energy-efficient performance of the R2WX system compared with the BDT system.

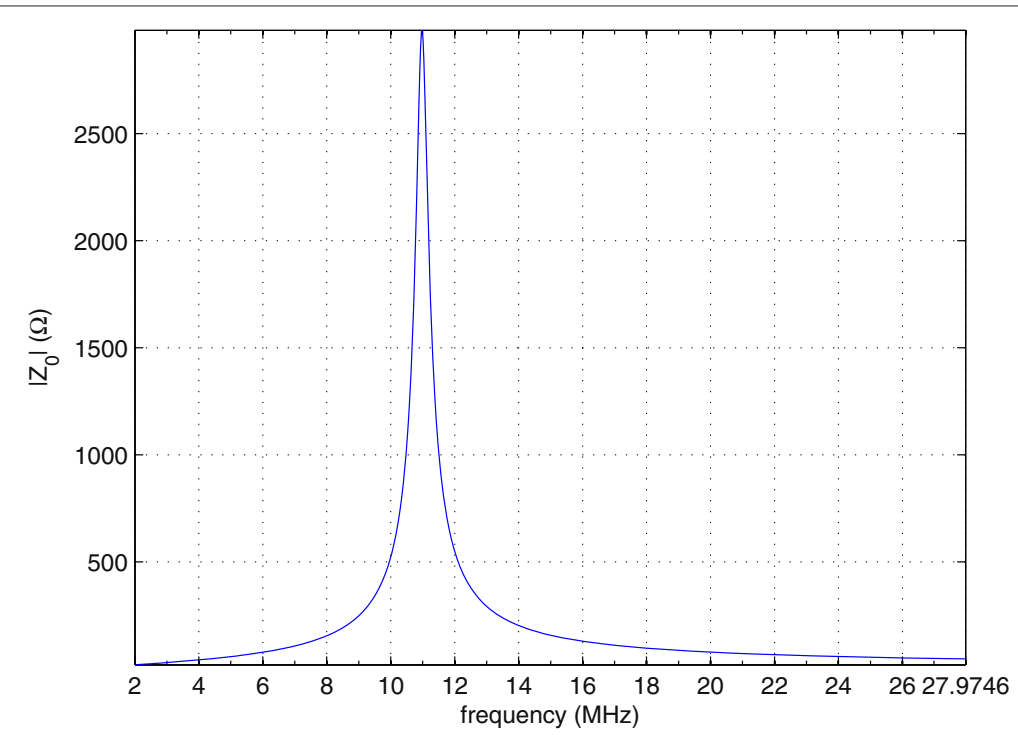

Fig. 8 Frequency response of preexisting load $Z_{0}$ 


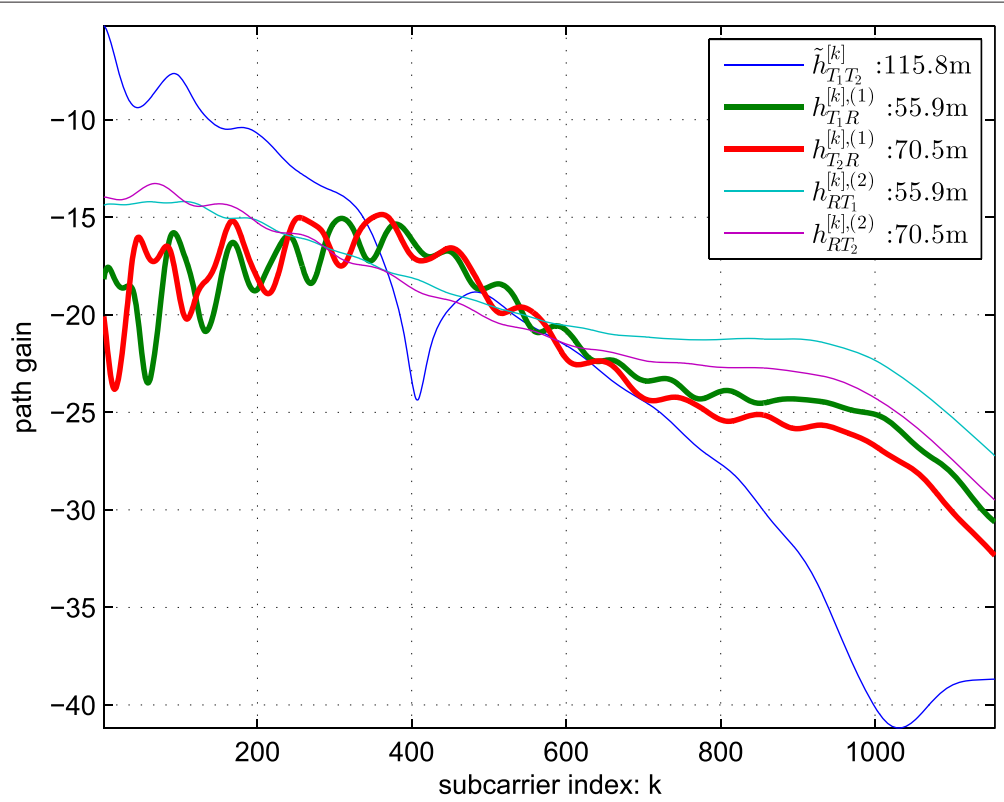

Fig. 9 Different path gains existing in the testing relay-involved channel where the P2P channel gain has also been given

Considering the possible EMI from broadband PLC systems to the shortwave radio systems, we hope this power saving property can relieve this issue to some extent.

\section{Conclusions}

We have developed an iterative algorithm to jointly optimize the power allocation between two terminals and one relay node for two-way multicarrier relay systems, where the two terminals exchange information in two signaling phases. In particular, we examined the minimization of the total transmission power when there are minimal capacity requirements on each traffic direction under the indoor PLC environment. Simulation results show that with respect to the conventional BDT system, the proposed algorithm can make the proposed R2WX system attain the same QoS requirements with less total transmission power.

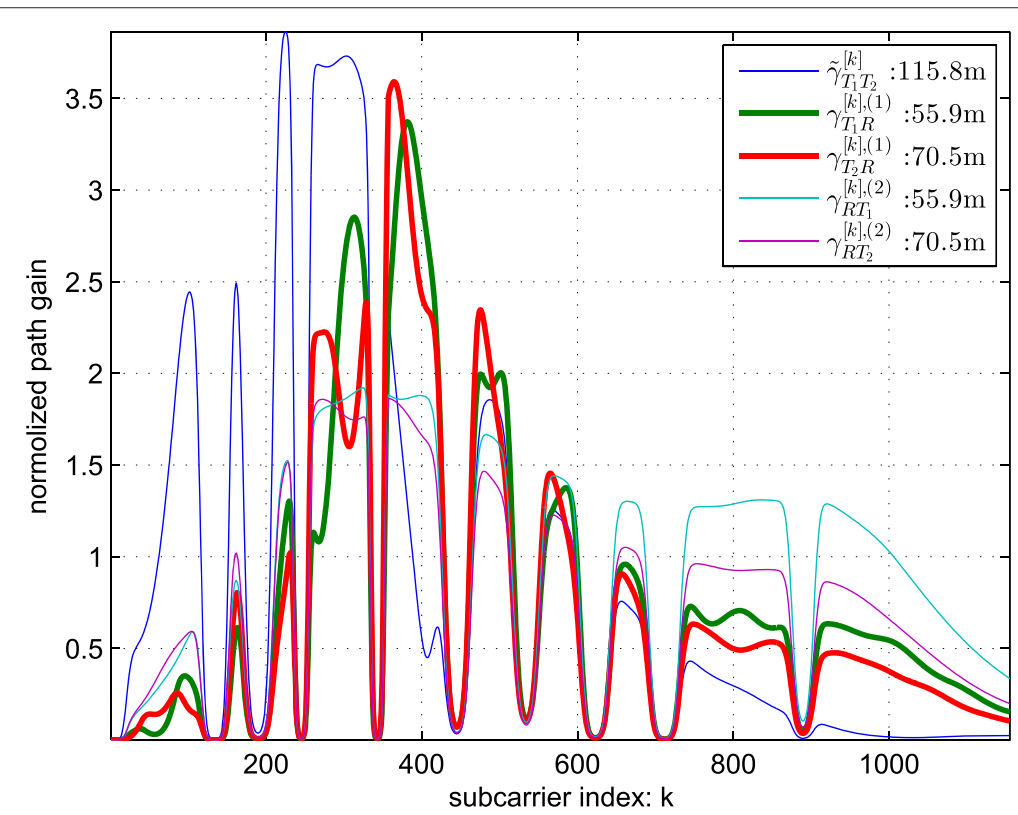

Fig. 10 Normalized gain of testing paths where the P2P channel normalized gain is also given 
Table 2 System parameters used for simulation

\begin{tabular}{ll}
\hline Frequency band & From 2 to $28 \mathrm{MHz}$ \\
\hline Total subcarriers number & $K=1155$ \\
General background noise & see Fig. 6 \\
Signaling path gains & see Fig. 9 \\
Normalized path gains & see Fig. 10 \\
\hline
\end{tabular}

\section{Appendix 1}

Lemma 1. Log-barrier $-\sum_{i=1}^{m} \log _{2}\left(f_{i}(x)\right)$ is convex on $\left\{x \mid f_{i}(x)>0\right\}$ if $f_{i}(x)$ is concave, where $m \in Z^{+}$[19].

Lemma 2. A non-negative weighted sum of convex (concave) functions is convex (concave) function [19].

Lemma 3. $-\log _{2} f(x)$ is quasi-convex, if $f(x)$ is quasiconcave on $\{x \mid f(x)>0\}[19]$.

Lemma 4. $\sum_{i=1}^{m} f_{i}(x)$ is quasi-convex (quasi-concave), if $f_{i}(x)$ is non-increasing (non-decreasing), where $m \in Z^{+}$ [19].

As $B_{k}, C_{k}, F_{k}, G_{k}>0$ and $D_{k}>1,\left(-\frac{B_{k}}{C_{k} x_{k}+D_{k}}\right)$ and $\left(-\frac{F_{k}}{G_{k} x_{k}+D_{k}}\right)$ in (25) and (26), respectively, are concave on $\left\{x_{k} \mid x_{k} \geq 0, k=1,2, \ldots, K\right\}$. From Lemmas 1 and 2 , it is easy to prove that the LHS of (25) and (26) are convex on $\left\{x_{k} \mid x_{k} \geq 0, k=1,2, \ldots, K\right\}$. Moreover, (24) and (27) are obviously convex. Thus, the problem (24)-(27) is convex on $\left\{x_{k} \mid x_{k} \geq 0, k=1,2, \ldots, K\right\}$.

Furthermore, it can be easily observed that the LHS of (35) is increasing with $x_{k}$, while the LHS of (36) and (37) are decreasing functions of $x_{k}$.

\section{Appendix 2}

As $H_{k}, J_{k}, O_{k}>0$ and $L_{k}, M_{k}, P_{k}>1$, the expression $\left(M_{k}-\frac{O_{k}}{J_{k} y_{k}+P_{k}}\right)$ in (42) is increasing and concave on $\left\{x_{k} \mid x_{k} \geq 0, k=1,2, \ldots, K\right\}$. From Lemma 1 , it is easy to prove that the LHS of (42) is convex on $\left\{x_{k} \mid x_{k} \geq 0, k=1,2, \ldots, K\right\}$.

The expression $\left(1+\frac{H_{k}}{J_{k} y_{k}+L_{k}}\right)$ in (41) is monotonically decreasing, so it is quasi-concave. From Lemmas 3 and 4 , the LHS of (41) is quasi-convex, so that the problem (40)-(43) is quasi-convex on $\left\{x_{k} \mid x_{k} \geq 0, k=1,2, \ldots, K\right\}$.

Furthermore, we can see that the LHS of (52) is decreasing with $y_{k}$, while the LHS of (50) and (51) are increasing functions of $y_{k}$.

\section{Appendix 3}

Let us write $x_{k}, y_{k}$, and $z_{k}(k=1,2, \ldots, K)$ in vector forms as $\mathbf{x}=\left[x_{1}, x_{2}, \ldots, x_{K}\right]^{T}, \mathbf{y}=\left[y_{1}, y_{2}, \ldots, y_{K}\right]^{T}$ and $\mathbf{z}=\left[z_{1}, z_{2}, \ldots, z_{K}\right]^{T}$, respectively, where $(\cdot)^{T}$ denotes the vector transpose.

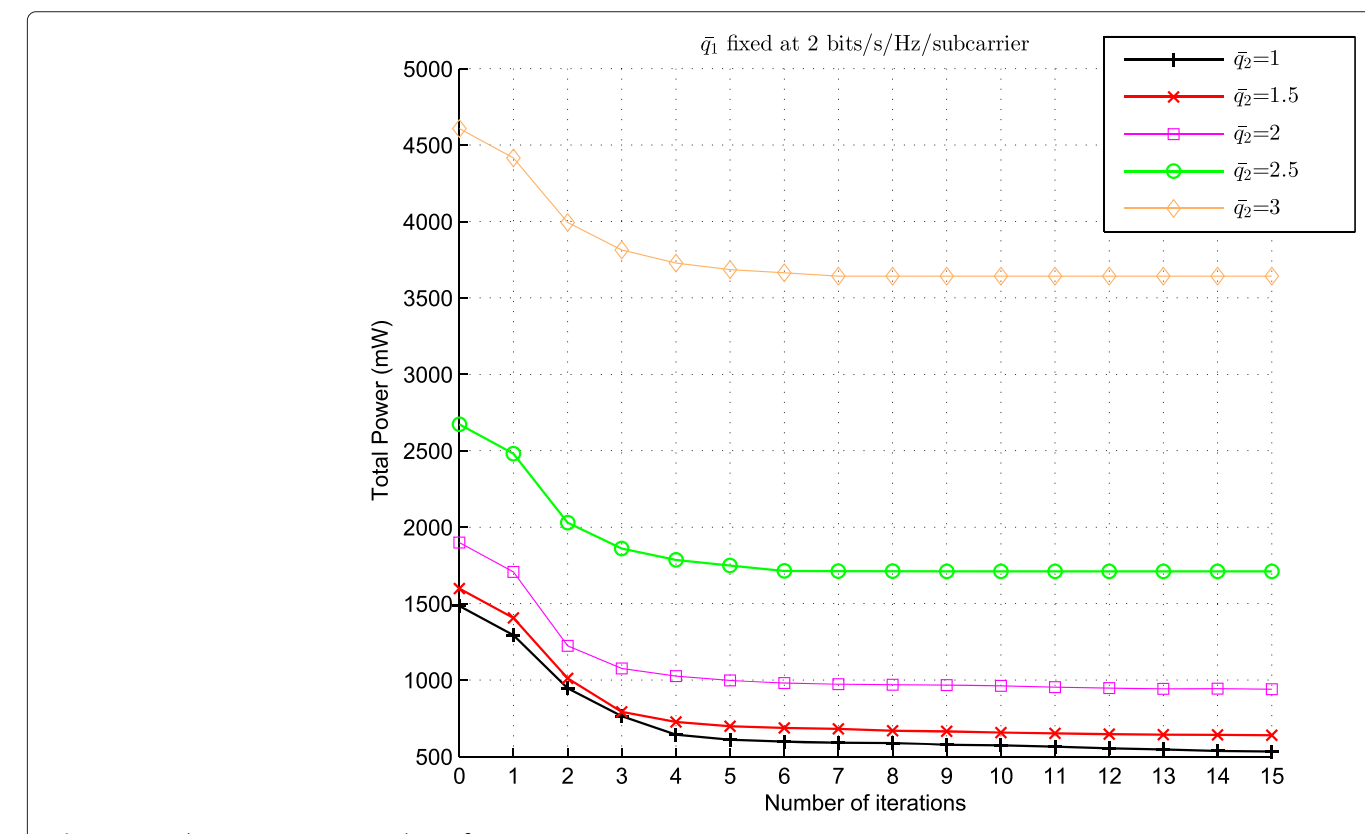

Fig. 11 Total power versus number of iterations 


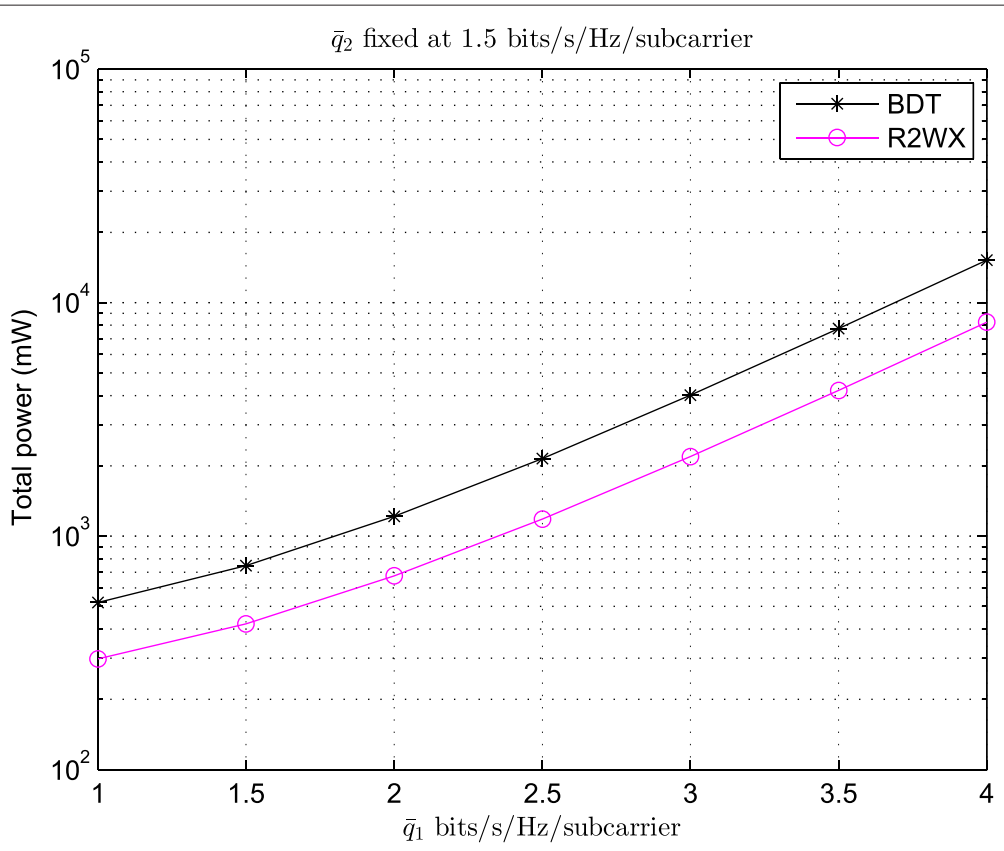

Fig. 12 Total power versus one directional ASC

Since all three sub-problems (24)-(27), (40)-(43), and (55)-(58) are convex/quasi-convex, the solution to each sub-problem is optimal. In other words, at a convergence point $\boldsymbol{\theta}^{\{n\}}=\left[\left(\mathbf{x}^{\{n\}}\right)^{T},\left(\mathbf{y}^{\{n\}}\right)^{T},\left(\mathbf{z}^{\{n\}}\right)^{T}\right]^{T}$, where the superscript $\{n\}$ denotes the variables at the $n$th iteration, we have

$$
\begin{aligned}
& \nabla_{\mathbf{x}} P\left(\boldsymbol{\theta}^{\{n\}}\right)^{T}\left(\mathbf{x}-\mathbf{x}^{\{n\}}\right) \geq 0 \\
& \nabla_{\mathbf{y}} P\left(\boldsymbol{\theta}^{\{n\}}\right)^{T}\left(\mathbf{y}-\mathbf{y}^{\{n\}}\right) \geq 0 \\
& \nabla_{\mathbf{z}} P\left(\boldsymbol{\theta}^{\{n\}}\right)^{T}\left(\mathbf{z}-\mathbf{z}^{\{n\}}\right) \geq 0 .
\end{aligned}
$$

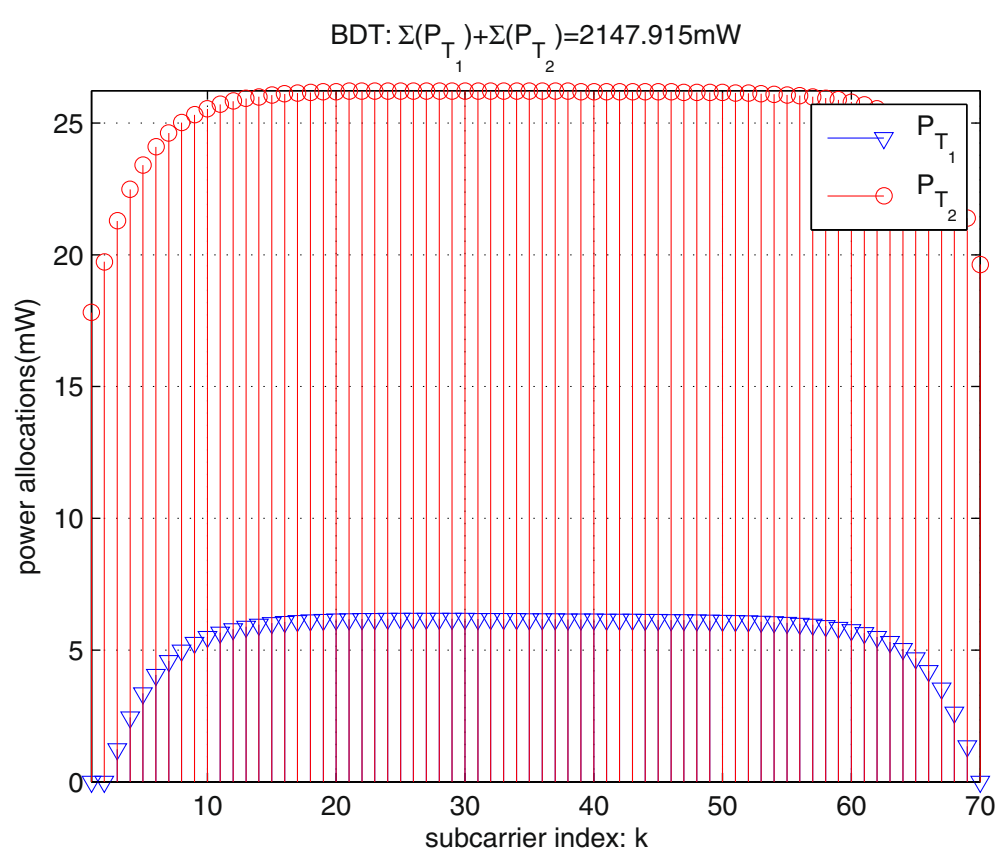

Fig. 13 BDT system 


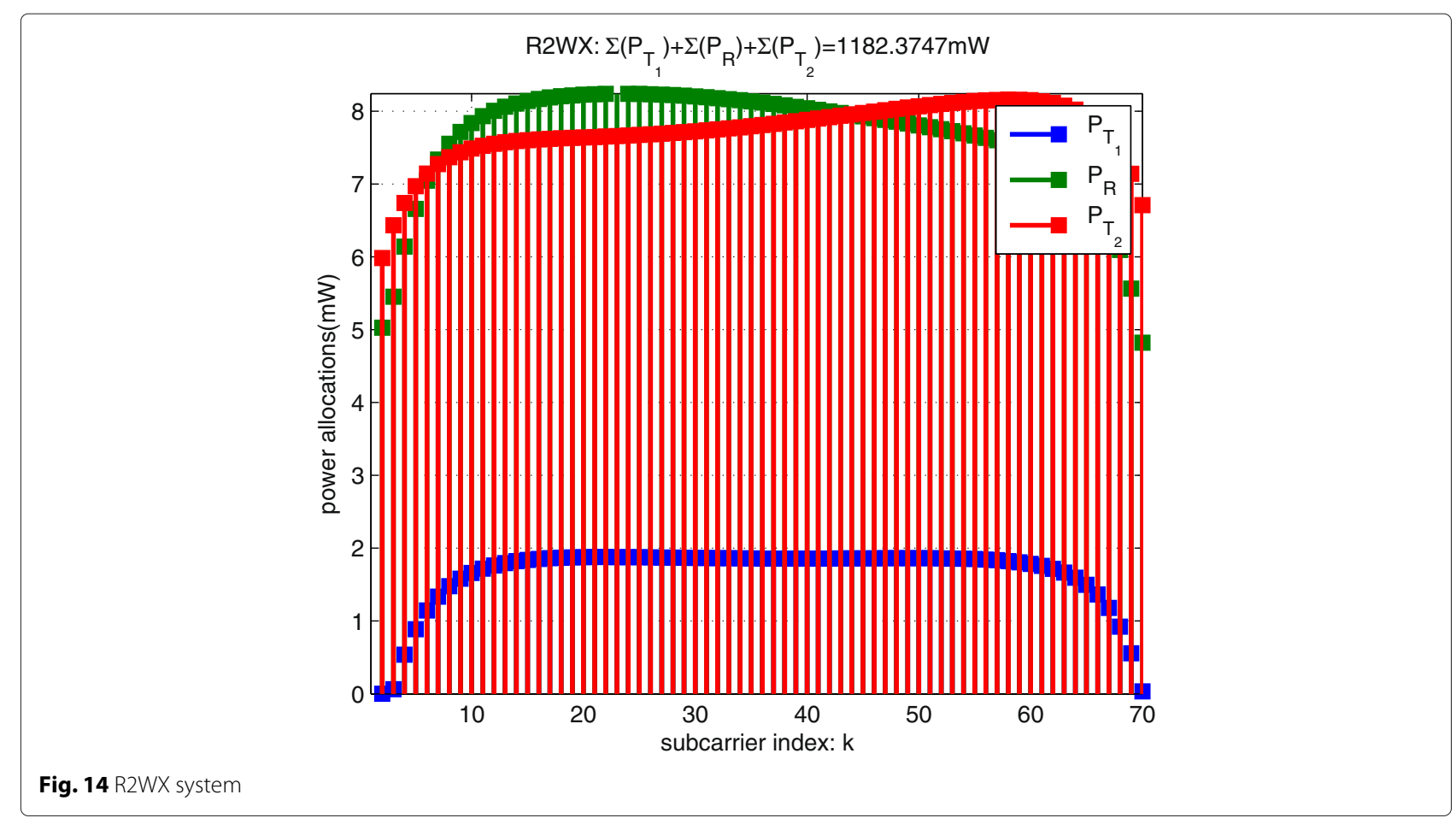

Here $\nabla_{\boldsymbol{\alpha}} P\left(\boldsymbol{\theta}^{\{n\}}\right)$ denotes the gradient of the objective function (15) along the direction of $\boldsymbol{\alpha} \in\{\mathbf{x}, \mathbf{y}, \mathbf{z}\}$ at $\boldsymbol{\theta}^{\{n\}}$. By summing up (71)-(73), we obtain

$$
\nabla P\left(\boldsymbol{\theta}^{\{n\}}\right)^{T}\left(\boldsymbol{\theta}-\boldsymbol{\theta}^{\{n\}}\right) \geq 0
$$

where $\nabla P\left(\boldsymbol{\theta}^{\{n\}}\right)=\left[\nabla_{\mathbf{x}} P\left(\boldsymbol{\theta}^{\{n\}}\right)^{T}, \nabla_{\mathbf{y}} P\left(\boldsymbol{\theta}^{\{n\}}\right)^{T}, \nabla_{\mathbf{z}} P\left(\boldsymbol{\theta}^{\{n\}}\right)^{T}\right]^{T}$. This indicates that $\boldsymbol{\theta}^{\{n\}}$ is a stationary point of (15).

\section{Competing interests}

The authors declare that they have no competing interests.

\section{Acknowledgements}

We thank our Editor and Reviewers for careful reading of our manuscript and helpful comments that significantly improve the quality of the manuscript.

Received: 25 January 2015 Accepted: 21 July 2015

Published online: 21 September 2015

\section{References}

1. S Galli, A Scaglione, Z Wang, For the grid and through the grid: The role of power line communications in the smart grid. Proc. IEEE. 99(6), 998-1027 (2011)

2. L Lampe, AH Vinck, in The Workshop on Power Line Communications (WSPLC). A study on relay network techniques in PLC (Doorwerth, The Netherlands, 2011)

3. L Lampe, AH Vinck, On cooperative coding for narrow band PLC networks. AEU-Int. J. Electron. Commun. 65(8), 681-687 (2011)

4. B Praho, M Tlich, P Pagani, A Zeddam, F Nouvel, in IEEE International Symposium on Power Line Communications and Its Applications (ISPLC), 2010. Cognitive detection method of radio frequencies on power line networks (IEEE, Rio de Janeiro, Brazil, 2010), pp. 225-230

5. T Sartenaer, P Delogne, in IEEE International Symposium on Power Line Communications and Its Applications (ISPLC). Powerline cables modelling for broadband communications (Udine, Italy, 2001), pp. 331-337
6. H Furukawa, H OKada, T Yamazato, M Katayama, in IEEE International Symposium on Power Line Communications and Its Applications (ISPLC). Signaling methods for broadcast transmission in power-line communication systems (Kyoto, Japan, 2003)

7. G Bumiller, in IEEE International Symposium on Power-Line Communications and Its Applications (ISPLC). Single frequency network technology for medium access and network management (Athens, Greece, 2002)

8. S D'Alessandro, AM Tonello, On rate improvements and power saving with opportunistic relaying in home power line networks. EURASIP J. Adv. Signal Process. 2012(1), 194 (2012)

9. L Lampe, R Schober, S Yiu, Distributed space-time coding for multihop transmission in power line communication networks. Selected Areas Commun. IEEE J. 24(7), 1389-1400 (2006)

10. JN Laneman, DN Tse, GW Wornell, Cooperative diversity in wireless networks: Efficient protocols and outage behavior. Inform. Theory, IEEE Trans. 50(12), 3062-3080 (2004)

11. S Katti, S Gollakota, D Katabi, in ACM SIGCOMM. Embracing wireless interference: Analog network coding (Kyoto, Japan, 2007)

12. CK Ho, R Zhang, Y-C Liang, in IEEE International Conference on Communications (ICC). Two-way relaying over OFDM: optimized tone permutation and power allocation (IEEE Beijing, China, 2008), pp. 3908-3912

13. B Tan, J Thompson, in IEEE International Conference on Communication (ICC) Workshops. Relay transmission protocols for in-door powerline communications networks (IEEE, Milan, Italy, 2011), pp. 1-5

14. M Noori, L Lampe, in IEEE Global Communications Conference (GLOBECOM). Improving data rate in relay-aided power line communications using network coding (IEEE, Atlanta, USA, 2013), pp. 2975-2980

15. N Papandreou, T Antonakopoulos, Bit and power allocation in constrained multicarrier systems: the single-user case. EURASIP J. Adv. Signal Process. 2008 (2008). 643081 doi:10.1155/2008/643081

16. $Y$ Rong, $X$ Tang, $Y$ Hua, A unified framework for optimizing linear nonregenerative multicarrier mimo relay communication systems. Signal Process. IEEE Trans. 57(12), 4837-4851 (2009)

17. S Galli, TC Banwell, in IEEE Consumer Communications and Networking Conference (CCNC). Modeling the indoor power line channel: new results and modem design considerations (IEEE, New York, USA, 2004), pp. 25-30

18. Y Li, W Wang, J Kong, W Hong, X Zhang, M Peng, in IEEE International Conference on Communications (ICC). Power allocation and subcarrier 
pairing in OFDM-based relaying networks (IEEE, Beijing, China, 2008), pp. $2602-2606$

19. S Boyd, L Vandenberghe, Convex Optimization. (Cambridge University Press, Cambridge, UK, 2004)

20. FJ Canete, JA Cortes, L Diez, JT Entrambasaguas, A channel model proposal for indoor power line communications. Commun. Mag. IEEE. 49(12), 166-174 (2011). doi:10.1109/MCOM.2011.6094022

21. Homeplug AV2, White Paper. http://www.homeplug.org/techresources/ resources/, Accessed on 17 Sep. 2015

22. JA Cortes, L Diez, FJ Canete, JJ Sanchez-Martinez, Analysis of the indoor broadband power-line noise scenario. Electromagnetic Compatibility, IEEE Trans. 52(4), 849-858 (2010). doi:10.1 109/TEMC.2010.2052463

23. D Benyoucef, in Proceedings of the 7th International Symposium on Power-Line Communications and Its Applications, Kyoto, Japan. A new statistical model of the noise power density spectrum for powerline communication, (2003), pp. 136-141

\section{Submit your manuscript to a SpringerOpen ${ }^{\circ}$ journal and benefit from: \\ - Convenient online submission \\ - Rigorous peer review \\ - Immediate publication on acceptance \\ - Open access: articles freely available online \\ - High visibility within the field \\ - Retaining the copyright to your article \\ Submit your next manuscript at $\boldsymbol{\triangleright}$ springeropen.com}

